\title{
Development of a user-friendly guideline for data analysis and sampling design strategy
}

\author{
Yvon Desnoyers $^{1, *}$ and Bart Rogiers ${ }^{2}$ \\ ${ }^{1}$ Geovariances, 49bis avenue Franklin Roosevelt, 77210 Avon, France \\ 2 SCK•CEN | Belgian Nuclear Research Centre, Boeretang 200, 2400 Mol, Belgium
}

Received: 23 October 2019 / Received in final form: 20 January 2020 / Accepted: 27 January 2020

\begin{abstract}
Within the H2020 INSIDER project, the main objective of work package 3 (WP3) is to draft a sampling guide for initial nuclear site characterization in constraint environments, before decommissioning, based on a statistical approach. The second task of WP3 aims at developing a strategy for sampling in the field of initial nuclear site characterization in view of decommissioning, with the most important goal to guide the end user to appropriate statistical methods (including, but not limited to those identified during the first overview task) to use for data analysis and sampling design. To aid the end user in applying this strategy, a user-friendly application for guiding the end user through the contents of the strategy and the initial characterization process is also developed.
\end{abstract}

\section{Introduction}

The EURATOM work program project INSIDER was launched in June 2017 (18 partners from 10 European countries). It aims at improving the management of contaminated materials arising from decommissioning and dismantling $(\mathrm{D} \& \mathrm{D})$ operations by proposing an integrated methodology of characterization. The methodology is based on advanced statistical processing and modelling, coupled with adapted and innovative analytical and measurement methods, in line with sustainability and economic objectives.

The overall objective of INSIDER is to develop and validate a new and improved integrated characterization methodology and strategy during the D\&D process, based on three main use cases:

- A nuclear R\&D facility: radioactive liquid and sludge in tank at JRC Ispra (Italy)

- A nuclear power plant: activated bio-shield concrete of the $\mathrm{BR} 3$ reactor (Belgium)

- A post accidental site remediation: contaminated soils beneath a CEA building (France).

INSIDER's activities are divided into 7 Work Packages, each targeting a specific objective (Fig. 1).

The main objective of Work Package 3 (WP3) is to draft a sampling guide for initial nuclear site characterization in constraint environments before decommissioning, based on a statistical approach. This is done by selecting state-of-the-art techniques concerning sampling design optimization, using prior information and multiple iterations, testing the approach through different case studies

\footnotetext{
* e-mail: desnoyers@geovariances.com
}

and reviewing the feedback from overall uncertainty calculations. The process followed to meet the main WP3 objective consists of four steps:

- Status: provide an overview of the available sampling design methods and state-of-the-art statistical techniques.

- Development: develop a strategy/methodology that makes use of state-of-the-art techniques, and present it in a user-friendly software application.

- Implementation: apply the methodology to the different test cases considered in order to test its adequacy.

- Guidance: summarize all the findings in a comprehensive sampling strategy guide.

This paper aims to present and share the mid-term outputs of WP3, in particular for the second task dealing with the development of a user-friendly guideline for data analysis and sampling design strategy.

\section{Sampling strategy development}

This second task of WP3 aims at developing a strategy for sampling in the field of initial nuclear site characterization in view of decommissioning, with the most important goal to guide the end user to appropriate statistical methods (including, but not limited to those identified during the first task [1]) to use for data analysis and sampling design. The first output of this second task is consequently a detailed report [2] that is summarised in the next sections.

\subsection{Overall strategy}

While the data analysis and sampling design methods that can be applied depend strongly on the situation and specific 


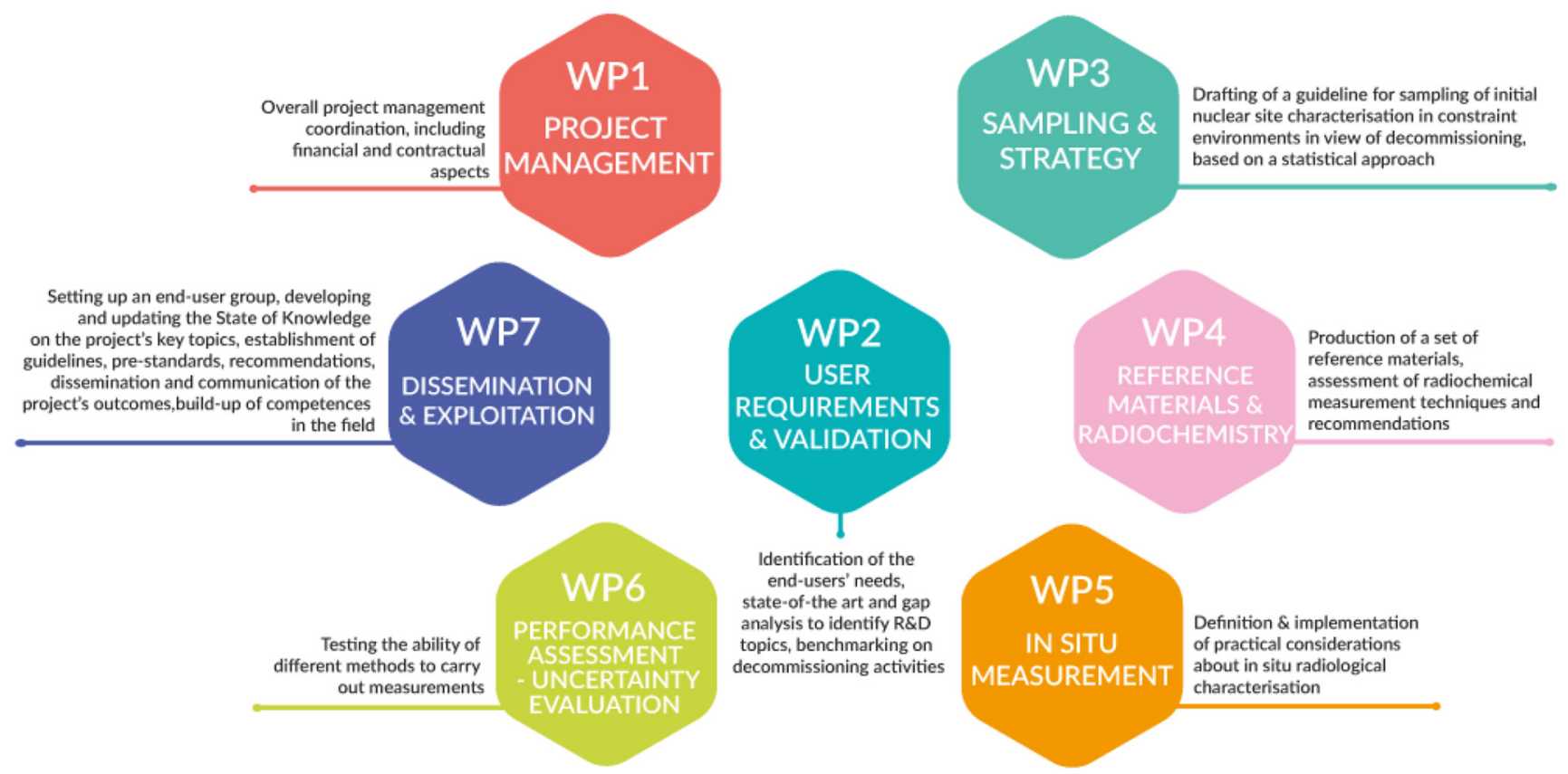

Fig. 1. INSIDER work package distribution.

goals of initial nuclear site characterization, the overall strategy often takes the form of the generic workflow illustrated in Figure 2.

The starting point considered here is the request for initial nuclear site characterization to a radiological characterization team. Such a request can come from different kinds of actors, and can come with different amounts of detail. Following this request, a clear list of all objectives and identification of the constraints is absolutely required, and might ask for some iterations with the applicant to agree on the goals and priorities. The highestpriority objective should be tackled first in most cases, and the cycle along the different objectives is started.

All prior information that is available and relevant for the investigated case should be gathered as a first step. If some data would already be available, a first analysis to check if the objective is achieved is probably very useful, even if the results come with lots of uncertainty. In D\&D, such prior information is nearly always available. Work is carried on historical installations and/or sites that have been shut down, or are going to be. Therefore, there is always a history of the exploitation phase, with available data, so this initial data-gathering step is of vital importance.

The data analysis following the data collection consists, in general, of the following steps: pre-processing, exploratory data analysis, the actual data analysis, and potentially a postprocessing step. If the objective is not achieved, a sampling design should be proposed using the most appropriate method(s) given all prior information and the data analysis result. Following the design, the corresponding characterization campaign should be performed. Additional characterization can reveal unexpected issues, and often revisiting the gathering of prior information is then useful. After the additional characterization, the updated dataset is again analysed, and the iterative procedure is continued until the objective is finally reached. The entire process can then be repeated to tackle the remaining objectives. Once all objectives have been achieved, the initial characterization study should be reported in a transparent way, making clear what has been measured, which results were obtained from the data analysis, and how large the corresponding uncertainty is.

\subsection{Data analysis}

For organizing the different data analysis techniques, the Venn diagram presented in Figure 3 is developed. The different categories are based on four aspects of the data, studied in the exploratory data analysis step:

- the requirement for multivariate methods to account for correlations between variables,

- the presence of spatial structure (non-randomness of spatial activity distribution),

- the presence of spatial trends (to be prior modelled possibly),

- and the requirement for robust methods (in case of small datasets).

The methods that are able to handle two, three or all aspects, are listed in the corresponding intersections. It is also possible none of these aspects apply, in which case the methods are presented outside of the diagram. More details on the individual methods are available in [2].

\subsection{Sampling design}

If the objective cannot be achieved with the available data, more information is required, and a proper sampling design should be made before collecting new data. There exists a variety of different ways to approach this, and the main drivers here are the available data, the type of problem at 


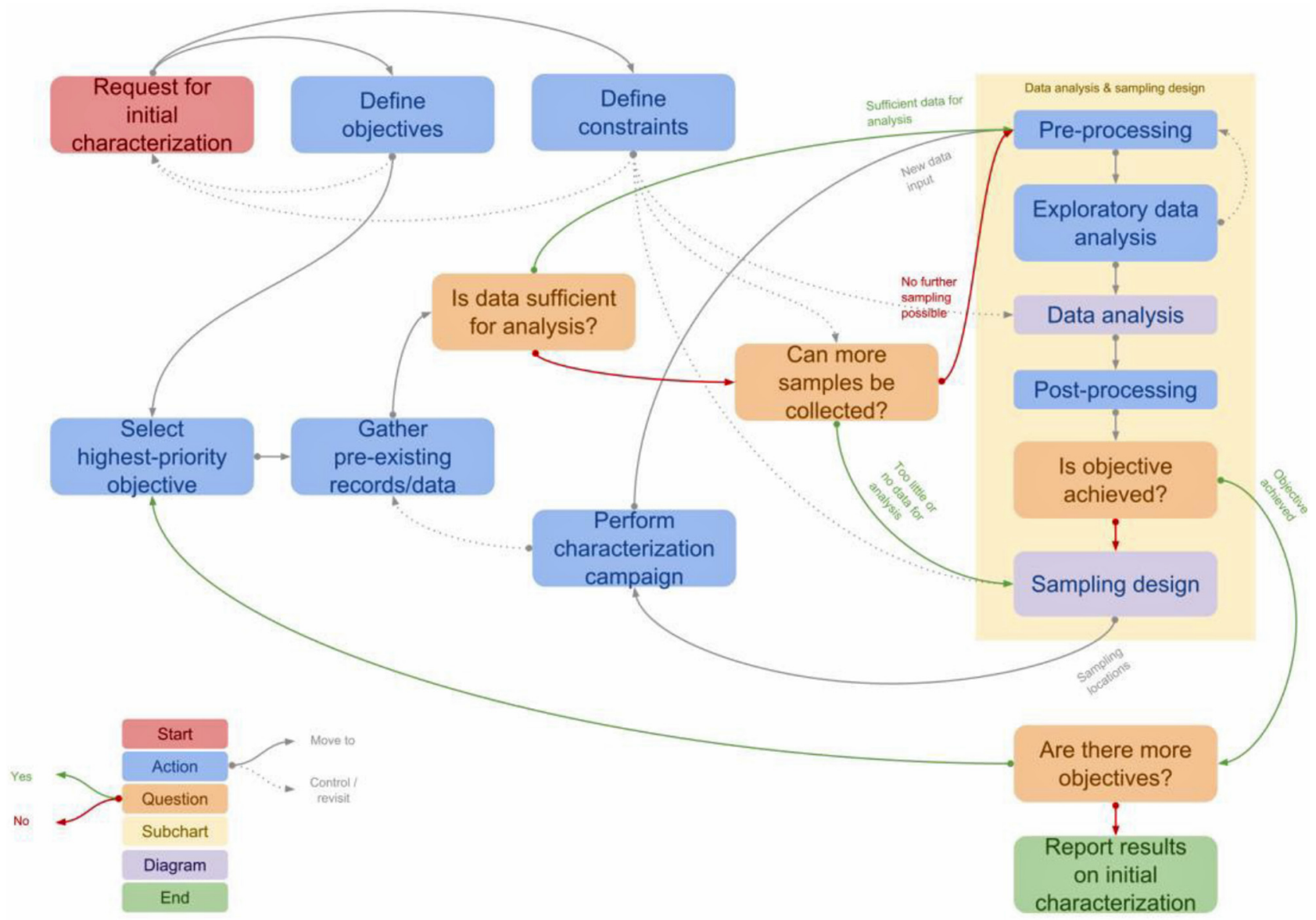

Fig. 2. Overall flowchart for sampling strategy and data analysis.

hand (revealed by the exploratory data analysis), the outcome of the data analysis, and the reason why the objective cannot be achieved. A similar Venn diagram organizes the selected sampling approaches according to their probabilistic or judgmental basis on the one hand, and an equal or unequal probability of selection on the other (Fig. 4). Note that the list of approaches provided here is non-limitative. Again, more details on the individual approaches are available in [2].

It should be noted here however, that in practice, sampling design consists most often of a combination of these approaches, as objectives and/or sampling targets often have multiple facets in real life.

\subsection{Optimisation}

Sampling strategy sometimes evolves into an iterative or adaptive approach. Based on a first sampling data set, it can be necessary to collect additional points in order to improve the initial estimation and/or to reduce related uncertainties. This sampling optimization is then strongly impacted by the characterization objective and can follow different rules.

- Statistics: add random points to improve statistics.

- Spatial clustering: add points around initial values that exceed a threshold (or any other criterion) to improve delineation.
- General optimisation: find the best set (number and location) of additional points using computer algorithms (simulated annealing, genetic algorithm...) for a given objective function.

\section{Implementation in a user-friendly interface}

To aid the end user in applying this strategy, a userfriendly application [3] for guiding the end user through the contents of the strategy and the initial characterization process is available online at https://insider-h2020. sckcen.be/. It has the same objectives:

- Define requirements for a statistical approach in the field of initial nuclear site characterisation in view of decommissioning combination of various non-destructive and destructive measurement results, sampling representability, multi variate analysis, overall associated uncertainties, accounting for prior knowledge.

- Help the user to select and develop an optimal statistical approach to be used in constraint environments.

\subsection{Used tools}

This deliverable is developed using $\mathrm{R}$ [4] and RStudio [5] and the following contributed $\mathrm{R}$ packages: 


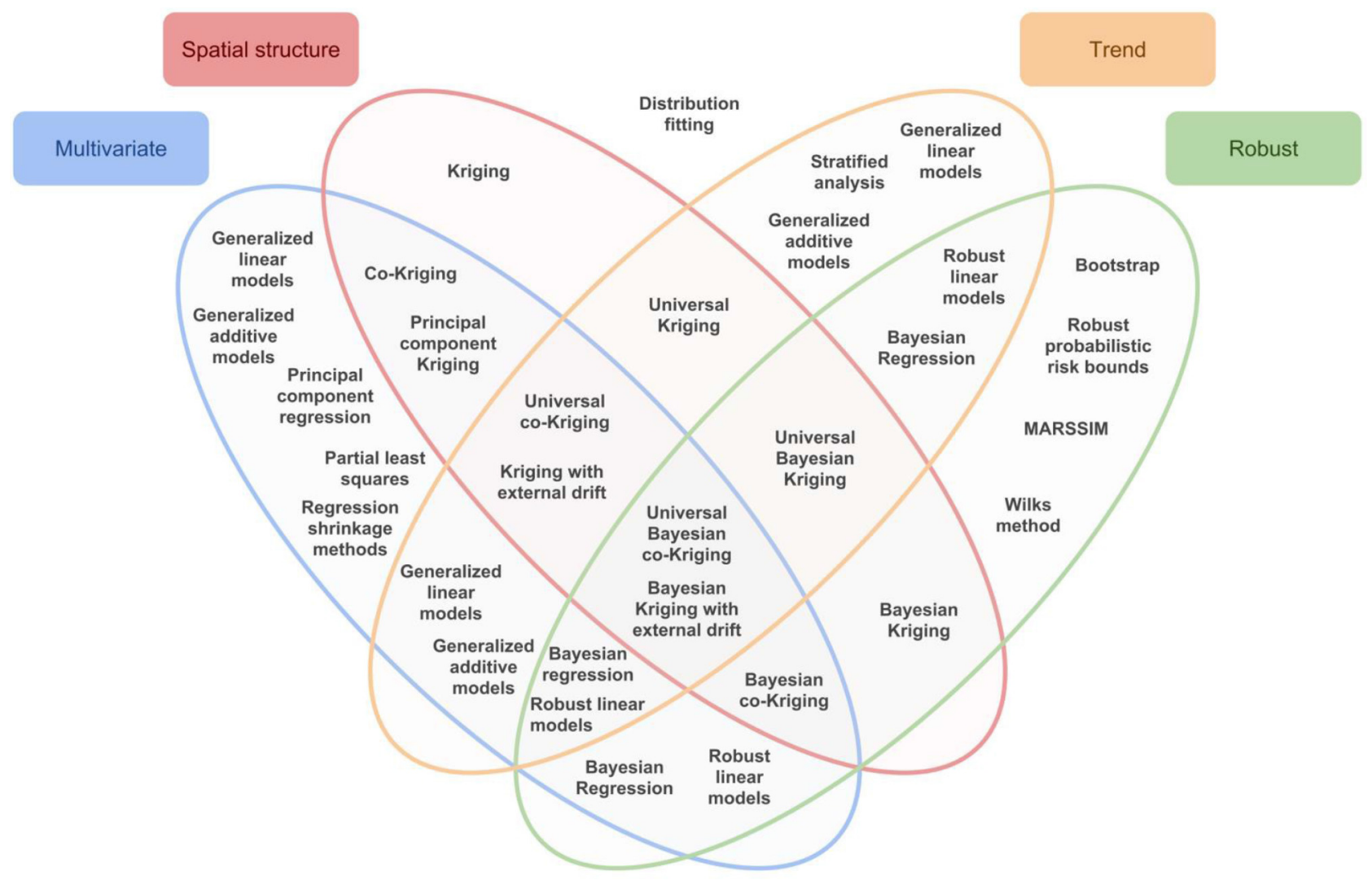

Fig. 3. Data analysis Venn diagram.

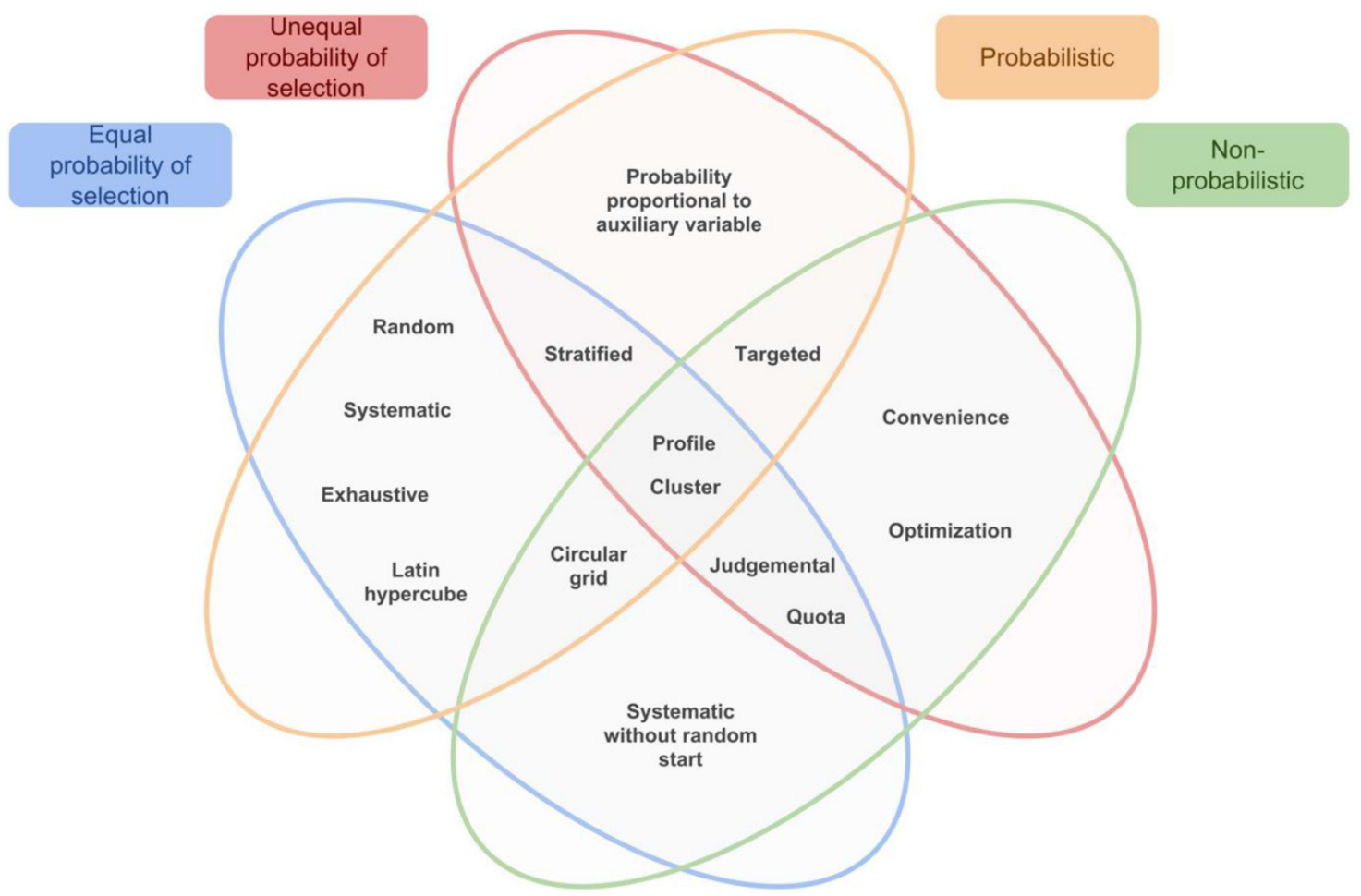

Fig. 4. Sampling design Venn diagram. 


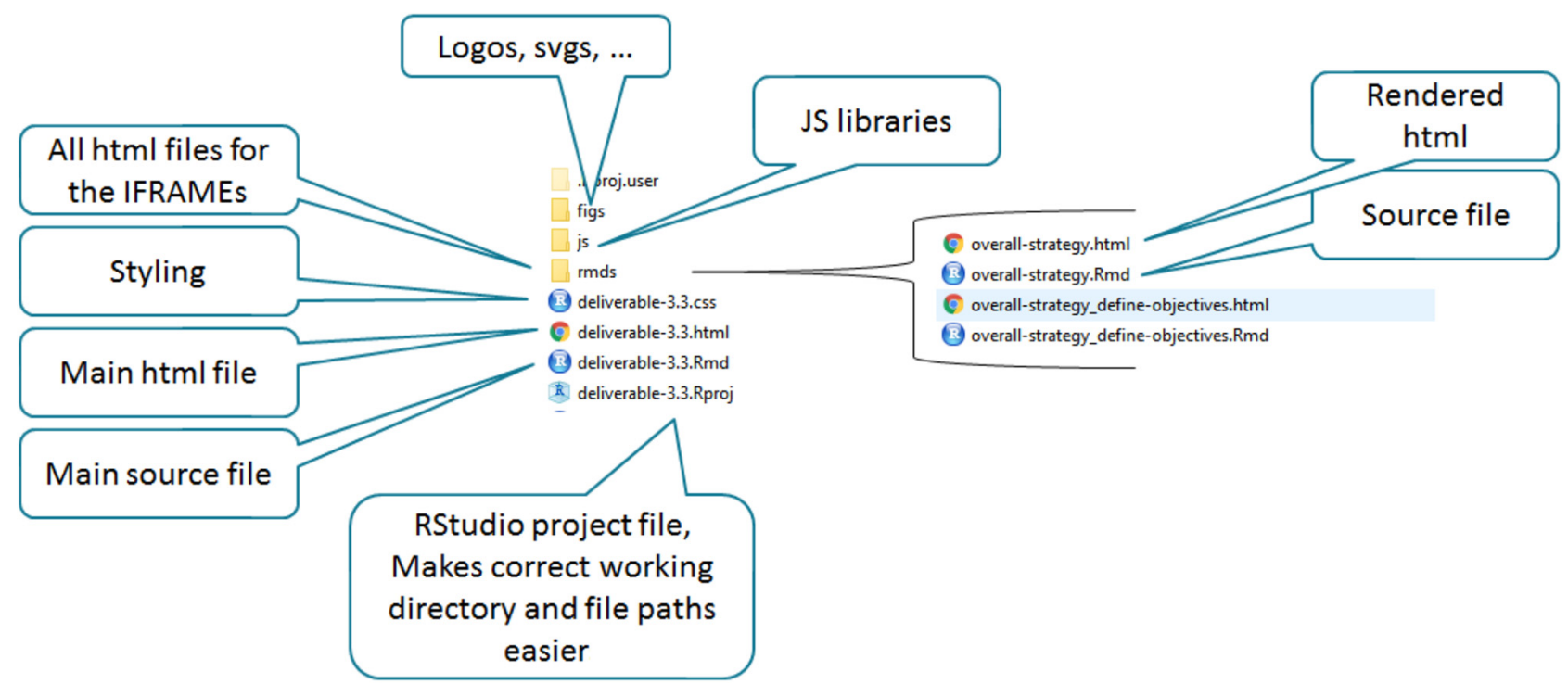

Fig. 5. File structure of the web-based interface.

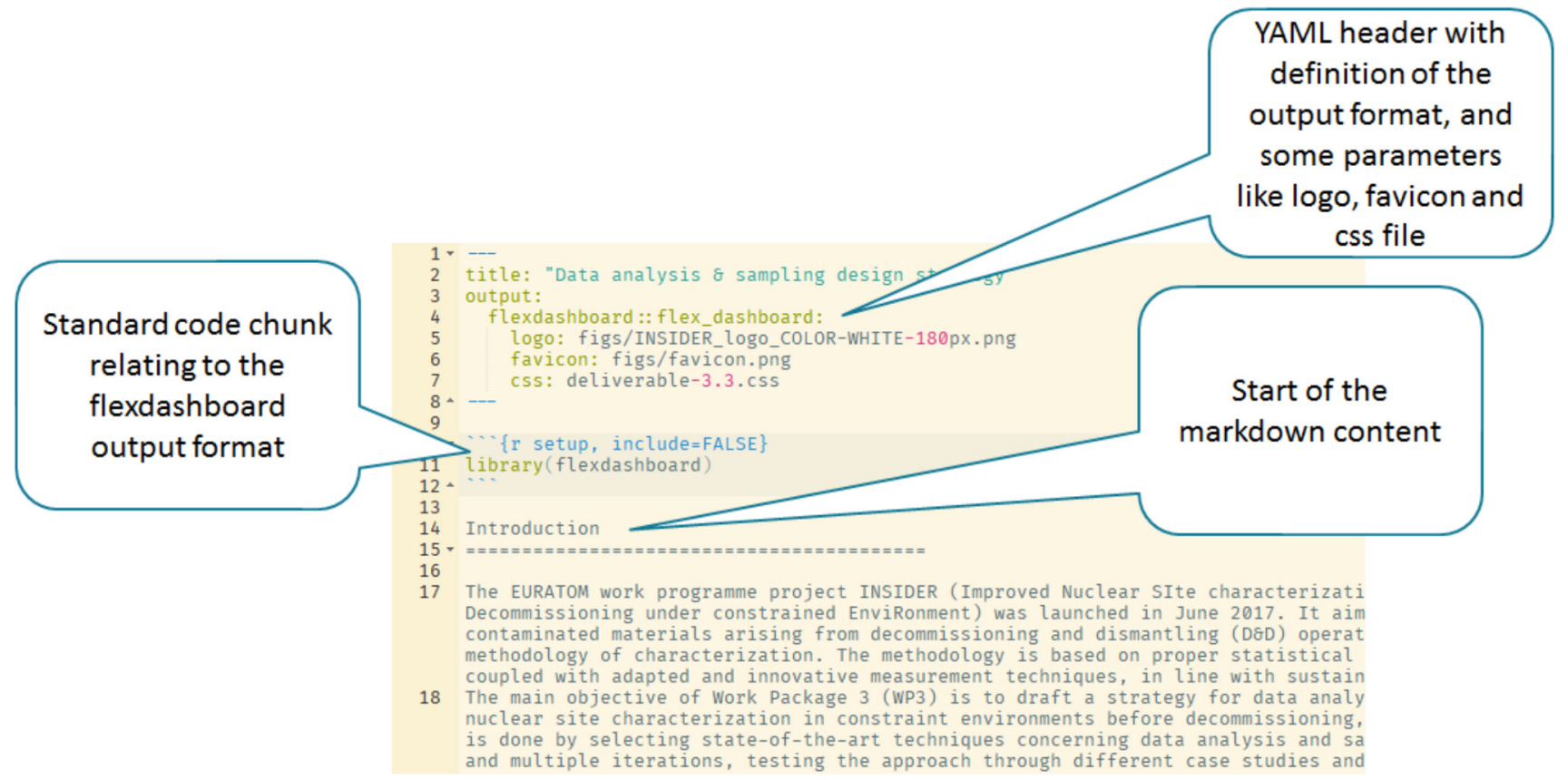

Fig. 6. Main *.Rmd file.

R Markdown [6]

- Allows writing in the simple markdown format (almost text files with specific header and easy formatting)

- Outputs are classical html files + JavaScript for an interactive website

- Flexdashboard [7]

- Provides a specific output format for the rmarkdown package

- Nice html + JavaScript dashboard for interactive apps svgPanZoom [8]

- Wrapper for svg-pan-zoom.js (https://github.com/ ariutta/svg-pan-zoom)

- Easily applied to SVGs from within R through the html widgets framework (https://www.htmlwidgets.org/)
- pacman [9] and here [10] for more automated and reproducible setup.

\subsection{Source files}

The file structure is presented in Figure 5. Input files (*.Rmd) and output files $(*$.html) are at the same level (both in the main project folder and the "rmds" subfolder). Other JavaScript libraries, widgets and figures are located in additional dedicated subfolders.

An example of source file (*.rmd) is presented in Figure 6. 


\section{UNSIDER Data analysis \& sampling design strategy Introduction Strategy - About}

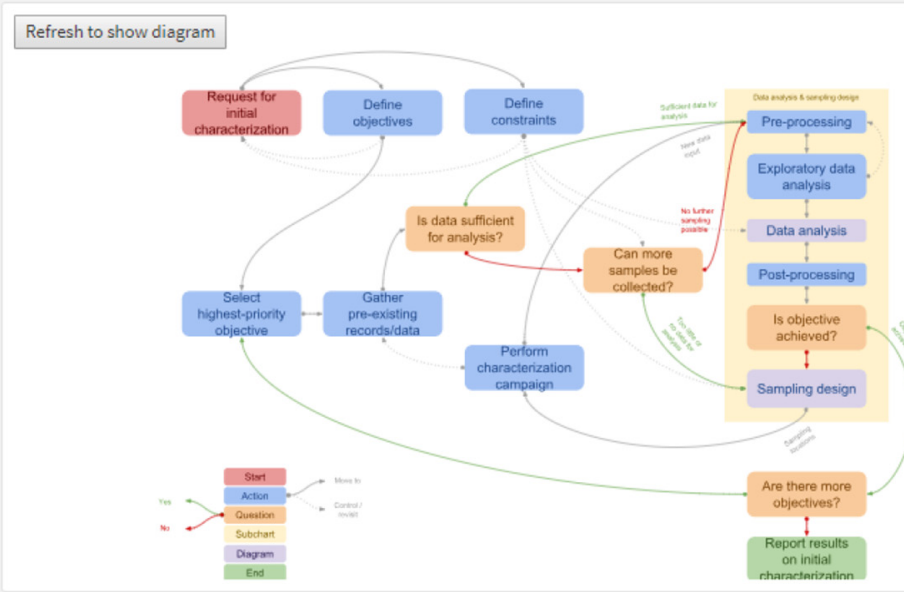

\section{Overall strategy}

While the data analysis and sampling design methods that can be applied depend strongly on the situation and specific goals of initial nuclear site characterization, the overall strategy often takes the form of the generic workflow illustrated in this flowchart.

The starting point we consider here is the request for initial nuclear site characterization to a radiological

characterization team. Such a request can come from different kinds of actors, and can come with different amounts of detail. Following this request, a clear list of all objectives and identification of the constraints is absolutely required, and might ask for some iterations with the requester to agree on the goals and priorities. The highest-priority objective should in most cases be tackled first, and the cycle along the different objectives is started.

Fig. 7. General view of the user-friendly interface to the strategy for data analysis and sampling design.

\section{INSIDER Data analysis \& sampling design strategy Introduction Strotegy - About}

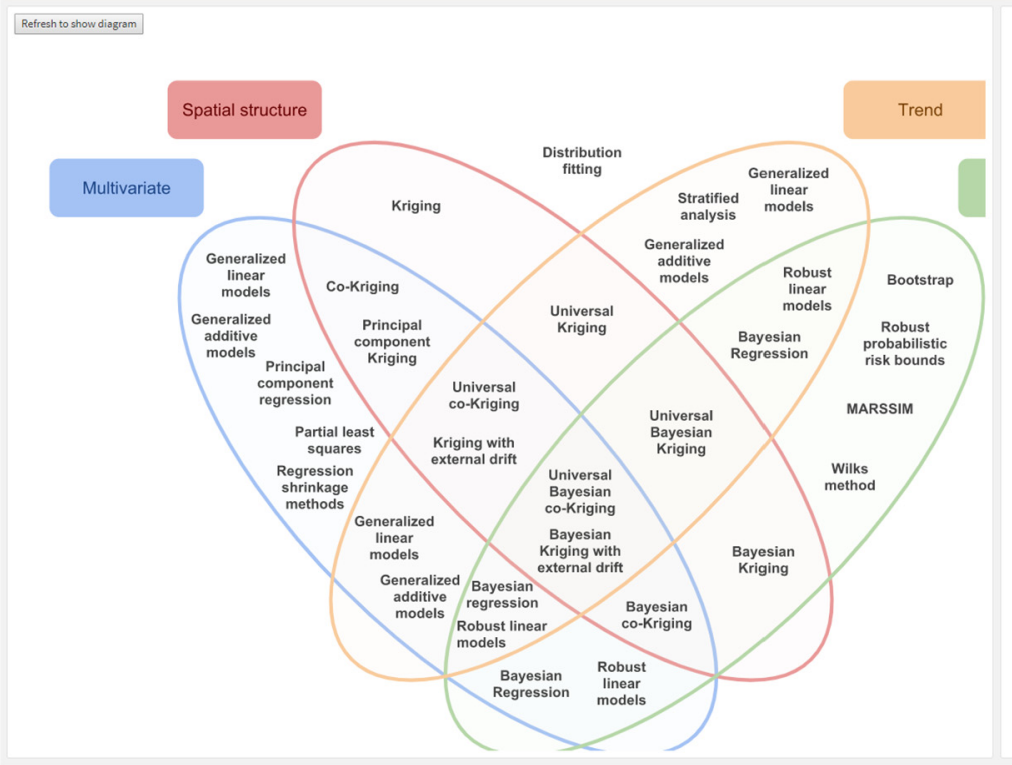

\section{Wilks method}

Workflow

The Wiks formula computes a quantle (or a tolerance interval) with a given confidence level from an i.i. sample, or computes the minimal sample size to estimate a quantle (or a tolerance interval) with a given confidence level This melhod based on order statisitics allows the user to determine the required sample size interest of this method is that it is robust and it requires no hypothesis Calculator: Wilks

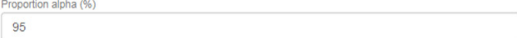

Probability beta (\%)

95

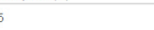

Example software implementations

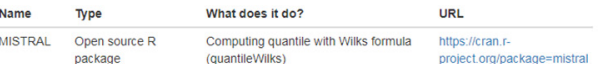

Example use cases

Reference Type

Blatman G, Delage T, looss B, Perot N. 2017. Probabilistic risk bounds Waste categorization \& cos for the characterization of radiological contamination. EPJ Nuclear Sci. estimation. H2 flow rate estimation

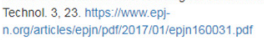
Pérot $N$. 2018. Sampling sirrategy for ditydrogen fow rate
characterization of racioactive waste, DEM 2018, Avignon.

Fig. 8. Example of a detailed page for data analysis (Wilks method), with the overview of methods in the Venn diagram.

\subsection{Overview of the resulting website}

The work consisted of the following actions:

- Convert text and tables of previous WP3 report [2] into R Markdown files

- Add links and targets to the different elements on the flow charts and Venn diagrams

- Knit/Render/Compile the ${ }^{*}$.Rmd files to get ${ }^{*}$.html output

- Possibly tweak some things in the resulting html files to get the desired behaviour (in particular additional JavaScript).

Example snapshots are presented in Figures 7 and 8.

\section{Conclusions and ongoing work}

In WP3 of the H2020 INSIDER project, the second task outlined a generic strategy for handling problem definition, data analysis and sampling design in the field of initial nuclear site characterization. Additionally, an overview of commonly used data analysis and sampling design methods, applicable in this field has been provided. This work served as a blueprint for the web-based application presenting the strategy in a more user-friendly way.

Furthermore, this approach is currently and thoroughly tested in practice within different use cases: 
- Use case 1: decommissioning of a back/end fuel cycle and/or research facility: radioactive liquid and sludge in tank at JRC Ispra (Italy).

- Use case 2: decommissioning of a nuclear reactor: activated bio-shield concrete of the BR3 reactor (Belgium).

- Use case 3: post accidental land remediation: contaminated soils beneath a CEA building (France).

The return-of-experience will allow refining the overall methodology for the final guideline developed within INSIDER WP3, describing the statistical approach and taking the uncertainty budget into consideration, potentially allowing further refinement of the web-based application in the final stage.

The INSIDER project received funding from the Euratom Research and Training Programme 2014-2018 under grant agreement No 755554.

\section{Author contribution statement}

The task leader within the INSIDER project is Bart Rodgiers. In particular, he worked on the global architecture of the interface and on the workflow diagrams. Yvon Desnoyers was mainly involved in the production of the different interface pages as well as the final compilation with javascript encapsulation.

\section{References}

1. N. Pérot, Y. Desnoyers, G. Augé, F. Aspe, S. Boden, B. Rogiers, O. Sevbo, O. Nitsche, INSIDER WP3-Sampling strategy - Report on the state of the art, Deliverable $3.1,2017$

2. B. Rogiers, S. Boden, N. Perot, Y. Desnoyers, O. Sevbo, O. Nitzsche, INSIDER WP3-Sampling strategy - Report on statistical approach, Deliverable D3.2, 2018

3. Y. Desnoyers, B. Rogiers, INSIDER WP3-Sampling strategy - Software of statistical approach, Deliverable D3.3, 2018

4. $\mathrm{R}$ Core Team, R: A language and environment for statistical computing. R Foundation for Statistical Computing, Vienna, Austria, 2019, available at https://www.Rproject.org/

5. RStudio Team, RStudio: Integrated Development for R. RStudio, Inc., Boston, MA, 2018, available at http://www. rstudio.com/

6. Y. Xie, J.J. Allaire, G. Grolemund, $R$ Markdown: The Definitive Guide (Chapman and Hall/CRC, New York, 2018)

7. R. Iannone, J.J. Allaire, B. Borges, flexdashboard: R Markdown Format for Flexible Dashboards. R package version 0.5.1.1, 2018, available at https://CRAN.R-project. org $/$ package $=$ flexdashboard

8. A. Riutta, J. Tangelder, K. Russell, svgPanZoom: R 'Htmlwidget' to Add Pan and Zoom to Almost any R Graphic. R package version $0.3 .3,2016$, available at https:// CRAN.R-project.org $/$ package $=$ svgPanZoom

9. T.W. Rinker, D. Kurkiewicz, pacman: Package Management for R. version 0.5.0. Buffalo, New York, 2017, available at http://github.com/trinker/pacman

10. K. Müller, here: A Simpler Way to Find Your Files. R package version 0.1, 2017, available at https://CRAN.Rproject.org $/$ package $=$ here

Cite this article as: Yvon Desnoyers, Bart Rogiers, Development of a user-friendly guideline for data analysis and sampling design strategy, EPJ Nuclear Sci. Technol. 6, 16 (2020) 